\title{
Erratum to: Non-synchronous and Localized Responses of Systems of Identical Centrifugal Pendulum Vibration Absorbers
}

\author{
Abdullah S. Alsuwaiyan · Steven W. Shaw
}

Published online: 18 December 2014

(c) King Fahd University of Petroleum and Minerals 2014

Erratum to: Arab J Sci Eng (2014) 39:9205-9217

DOI 10.1007/s13369-014-1464-1

The original version of this article unfortunately contains a mistake.

The correct form of Eq. (13) is:

$$
\begin{aligned}
a_{k}^{\prime}=\epsilon & {\left[-\frac{\tilde{\mu}_{a}}{2} a_{k}+\frac{\tilde{\Gamma}_{\theta}}{2 n} \sin \left(\phi_{k}\right)\right.} \\
& \left.+\frac{n}{2 N}(N-M) a_{l} \sin \left(\phi_{k}-\phi_{l}\right)\right]+\cdots \\
a_{k} \phi_{k}^{\prime}=\epsilon & {\left[-\frac{3 \gamma_{o}}{4 n} a_{k}^{3}+n\left(\sigma_{k}+\frac{M}{2 N}\right) a_{k}+\frac{\tilde{\Gamma}_{\theta}}{2 n} \cos \left(\phi_{k}\right)\right.} \\
& \left.+\frac{n}{2 N}(N-M) a_{l} \cos \left(\phi_{k}-\phi_{l}\right)\right]+\cdots
\end{aligned}
$$

The online version of the original article can be found under doi:10.1007/s13369-014-1464-1.

A. S. Alsuwaiyan $(\varangle)$

Department of Mechanical Engineering, Unayzah College of Engineering, Qassim University, Unayzah 51911, Saudi Arabia

e-mail: asaoiean@qu.edu.sa; suwaiyana@yahoo.com

\section{S. W. Shaw}

Department of Mechanical Engineering, Michigan State University, East Lansing, MI 48824-1226, USA

e-mail: shawsw@egr.msu.edu $a_{l}^{\prime}=\epsilon\left[-\frac{\tilde{\mu}_{a}}{2} a_{l}+\frac{\tilde{\Gamma}_{\theta}}{2 n} \sin \left(\phi_{l}\right)\right.$

$\left.+\frac{n}{2 N} M a_{k} \sin \left(\phi_{l}-\phi_{k}\right)\right]+\cdots$

$a_{l} \phi_{l}^{\prime}=\epsilon\left[-\frac{3 \gamma_{o}}{4 n} a_{l}^{3}+n\left(\sigma_{l}+\frac{(N-M)}{2 N}\right) a_{l}+\frac{\tilde{\Gamma}_{\theta}}{2 n} \cos \left(\phi_{l}\right)\right.$

$\left.+\frac{n}{2 N} M a_{k} \cos \left(\phi_{l}-\phi_{k}\right)\right]+\cdots$ 